\title{
The Electrophysiological Memory Function of Hippocampus Different from O'Keefe and Mosers
}

Zi-Jian Cai

No. 129, Building 6, Room 404, North Dongwu Road, Suzhou City, Jiangsu Province, 215128, PR China

*Corresponding author: Zi-Jian Cai, No. 129, Building 6, Room 404, North Dongwu Road, Suzhou City, Jiangsu Province, 215128 PR China, Tel: +86 512 65299403; E-mail: hrsh8@126.com

Received date: February 15, 2016; Accepted date: March 22, 2016; Published date: March 29, 2016

Copyright: (c) 2016 Cai ZJ. This is an open-access article distributed under the terms of the Creative Commons Attribution License, which permits unrestricted use, distribution, and reproduction in any medium, provided the original author and source are credited.

\begin{abstract}
The electrophysiology of hippocampus demonstrated by O'Keefe and Mosers is yet unattainable to explain the same memory function of mammillary bodies. The limbic-reticular coupling theory I proposed previously suggested the descending limbic system regulate the ascending reticular systems to consolidate and recall declarative memories. On electrophysiology, the hippocampal CA3 neurons can regulate the raphe nuclei and ventral tegmental area in their activities, while the cholinergic-mediated gamma wave is also coupled to theta activities. In this way, through these limbic-reticular electrophysiological couplings, the hippocampus can synchronously recall several components of a memory dispersed in various cortical areas. Therefore, here is outlined the alternative part of hippocampal electrophysiology on memory recall different from O'Keefe and Mosers.
\end{abstract}

Keywords: Hippocampus; Mammillary body; Acetylcholine; Dopamine; Theta wave; Gamma wave

\section{Communicated Opinion}

The 2014 Nobel Laureates O'Keefe and Mosers demonstrated with electrophysiology that the cells in entorhinal cortex and hippocampus directly encoded and associated information on place and direction [1] including those from the prefrontal cortex, with which to explain the memory function of hippocampal formation. However, this is an oversimplified explanation about the hippocampal role in human memory. First, the hippocampal amnesia manifests general rather than spatial memory impairment on declarative objects and events [2]. Second, alike to the hippocampal amnesia, the amnesia from lesions of either fornix or mammillary bodies also manifests the same memory impairment as deficit in recall $[3,4]$. Third, the orbitofrontal cortex manifests clinically irrelevant to memory recall $[5,6]$.

The limbic-reticular coupling theory suggests that the hippocampus and amygdala control and regulate such descending limbic structures as the mammillary bodies, septum, hypothalamus and epithalamus, and in turn regulate the ascending noradrenergic, serotonergic, dopaminergic and cholinergic systems to accomplish declarative memory consolidation and recall [2]. There are a lot of anatomical, neuropsychological, behavioral and pharmacological evidences in support of this theory [2].

On electrophysiology, the hippocampal CA3 activation has been shown to regulate the activity of serotonergic neurons in raphe nuclei $[7,8]$, while the hippocampal CA3 theta wave has been shown to activate the dopaminergic neurons in ventral tegmental area in contextual recall [9]. Besides, the cholinergic-mediated gamma wave has been shown to be modulated by theta activities from the medial temporal lobe $[10,11]$, and to be responsible for recalling memories in cortex [10-12]. In this way, through regulation on the ascending serotonergic, dopaminergic and cholinergic systems, the hippocampal CA3 neurons can synchronously recall several components of a memory dispersed in various cortical areas via theta-gamma coupling. This is the alternative story of hippocampal electrophysiology on memory recall which is different from O'Keefe and Mosers.

It is necessary to point out that the hippocampal activities demonstrated by O'Keefe and Mosers are activated directly by external cues, and are not the unretrieved signals waiting for hippocampus to recall. As believed by the world people, the unretrieved signals for hippocampus to recall are most likely present together with other signals within hippocampus, and are certainly most likely present as hippocampal CA3 theta activities.

It is noted that the noradrenergic and serotonergic systems stop discharge while the dopaminergic and cholinergic systems continue their activities during REM sleep which processes memories [13,14]. Decrease in serotonergic while maintenance in dopaminergic and cholinergic activities during REM sleep is alike to the above electrophysiological recall of memory.

In all, the limbic-reticular theta-gamma coupling is more appropriate to elucidation of the memory recall function from hippocampus to mammillary bodies, as the alternative part of hippocampal electrophysiology different from O'Keefe and Mosers.

\section{References}

1. Moser MB (2014) Nobel Lecture: Grid Cells, Place Cells and Memory. Nobelprize.org, Nobel Media AB.

2. Cai ZJ (1990) The neural mechanism of declarative memory consolidation and retrieval: a hypothesis. Neurosci Biobehav Rev 14: 295-304.

3. Tsivilis D, Vann SD, Denby C, Roberts N, Mayes AR, et al. (2008) A disproportionate role for the fornix and mammillary bodies in recall versus recognition memory. Nat Neurosci 11: 834-842.

4. Carlesimo GA, Serra L, Fadda L, Cherubini A, Bozzali M, et al. (2007) Bilateral damage to the mammillo-thalamic tract impairs recollection but not familiarity in the recognition process: a single case investigation. Neuropsychologia 45: 2467-2479. 
Citation: Cai ZJ (2016) The Electrophysiological Memory Function of Hippocampus Different from O'Keefe and Mosers. J Neurol Neurophysiol 7: 362. doi:10.4172/2155-9562.1000362

Page 2 of 2

5. Fujii T, Suzuki M, Suzuki K, Ohtake H, Tsukiura T, et al. (2005) Norma memory and no confabulation after extensive damage to the orbitofrontal cortex. J Neurol Neurosurg Psychiatry 76: 1309-1310.

6. Stuss DT, Kaplan EF, Benson DF, Weir WS, Chiulli S, et al. (1982) Evidence for the involvement of orbitofrontal cortex in memory functions: an interference effect. J Comp Physiol Psychol 96: 913-925.

7. Vinogradova OS (2001) Hippocampus as comparator: role of the two input and two output systems of the hippocampus in selection and registration of information. Hippocampus 11: 578-598.

8. Varga V, Kekesi A, Juhasz G, Kocsis B (1998) Reduction of the extracellular level of glutamate in the median raphe nucleus associated with hippocampal theta activity in the anaesthetized rat. Neuroscience 84 : 49-57.

9. Luo AH, Tahsili-Fahadan P, Wise RA, Lupica CR, Aston-Jones G (2011) Linking context with reward: a functional circuit from hippocampal CA3 to ventral tegmental area. Science 333: 353-357.
10. Kaplan R, Bush D, Bonnefond M, Bandettini PA, Barnes GR, et al. (2014) Medial prefrontal theta phase coupling during spatial memory retrieval. Hippocampus 24: 656-665.

11. Foster BL, Kaveh A, Dastjerdi M, Miller KJ, Parvizi J (2013) Human retrosplenial cortex displays transient theta phase locking with medial temporal cortex prior to activation during autobiographical memory retrieval. J Neurosci 33: 10439-10446.

12. Foster BL, Dastjerdi M, Parvizi J (2012) Neural populations in human posteromedial cortex display opposing responses during memory and numerical processing. Proc Natl Acad Sci USA 109: 15514-15519.

13. Cai ZJ (1995) An integrative analysis to sleep functions. Behav Brain Res 69: 187-194.

14. Cai ZJ (2015) Extending psychoanalysis with theories on sleep functions. J Sleep Disord Ther 4: 217. 